\title{
CIANOFÍCEAS DE SOLO E CALÇAMENTO NO CAMPUS DA UNIVERSIDADE FEDERAL DE SANTA MARIA, RIO GRANDE DO SUL, BRASIL.
}

\author{
Marlene Pilecco Bertagnolli \\ Aluna do Curso de Especialização em Biologia \\ João Fernando Prado \\ Departamento de Biologia - Centro de Ciências Naturais e Exatas \\ U.F.S.M. - Santa Maria, RS
}

RESUMO

O trabalho consta de um levantamento e enquadramento taxonômico dos gêneros de cianoficeas de solo e calçamento, encontrados em 5 estações, localizadas na área do Campus da Universidade Federal de Santa Maria. Foram realizadas 8 coletas, no decorrer dos anos de 1992 e 1993, utilizando-se o método de raspagem de substratos, obtendo-se 22 gêneros distribuídos em 5 familias e 3 ordens.

\section{SUMMARY}

The work consists in a taxonomic survey and classification of genera of bluegreen algae from soil and paving, which were found in eight samplis and conducted in five stations in the Campus or Universidade Federal de Santa Maria. Twenty two genera were obtained and distributed in five families and three orders. 
Palavras chaves: cianoficeas terrestres, cianoficeas de solo e sistemática

\section{INTRODUÇÃO}

Com uma organização bastante primitiva, as cianoficeas são consideradas os procariontes fotossintetizantes mais antigos. Apresentam uma grande amplitude ecológica, ocupando os mais variados ambientes.

No solo, além de aumentarem em 30 a $38 \%$ o conteúdo de nitrogênio, pela fixação do $\mathrm{N}_{2}$ atmosférico ( $\mathrm{ROUND}, 1983$ ), colaboram na produção de humus, preparando o substrato para o desenvolvimento de outras plantas ( SENNA, 1982 ); atuam na colonização, iniciando a sucessão de comunidades ( SHI-MEI e SHANG-HOO, 1991 ); previnem a erosão pelo entrelaçamento dos filamentos que ligam as partículas de solo, mantendo um maior conteúdo de água ( DANIN et al.,1989; KOSTYKOV, 1990;,DAVEY et al., 1991 ); podem ser utilizadas como fertilizantes, pois acrescentam substâncias químicas, tanto por sua morte quanto por difusão a partir das células ( ROUND, 1983 ). Nas dunas, as cianoficeas filamentosas diminuem a velocidade de deslocamento, tanto pelo entrelaçamento de seus filamentos, quanto pela mucilagem excretada por seus tricomas, prendendo os grãos de areia ( DANIN et al., 1989 ). Atribui-se também a elas a formação do solo através da deterioração das rochas ( ORTEGA; HERNANDEZ; SAIZ, 1991 ) e certas espécies decompõem silicatos que originam alguns tipos de solo ( SENNA, 1982 ).

No Brasil, trabalhos relativos à cianoficeas de solo, foram feitos por LYRA (1972), OLIVEIRA et al. ( 1980 ), SENNA ( 1982 ) e AZEVEDO ( 1991 ). Em relação à cianoficeas de calçamento, não foram encontrados estudos.

Este trabalho é parte da monografia apresentada ao curso de Especialização em Biologia da U.F.S.M. e visa o levantamento de gêneros de cianoficeas de solo e calçamento, obtidas de análises do material encontrado nas 45 amostras que fazem parte de 8 coletas realizadas no campus da Universidade Federal de Santa Maria, como forma de contribuição ao conhecimento da flórula ficológica de Santa Maria e, conseqüentemente, do Rio Grande do Sul e do Brasil.

\section{LOCAL DE ESTUDO}

Na cidade de Santa Maria, situada no centro geográfico do Rio Grande do Sul

(Fig. 1) e enquadrada entre as coordenadas de 5330'22" e 5419'32" de longitude Oeste e 
2920'28" e 3000'16"de latitude Sul (PEREIRA et. al. 1989) está localizado o campus da Universidade Federal de Santa Maria. O campus tem uma área de 1.121,14 ha (Fig.2) e faz parte do bairro de Camobi. Na área do campus foram estabelecidas 5 estações de coletas (Fig 3) nos dois tipos seguintes de habitats:

- solo - refere-se à superficie do terreno, recoberta ou não por gramado.

- calçamento - refere-se a lages e lajotas dos passeios públicos e paralelepípedos das ruas e avenidas.

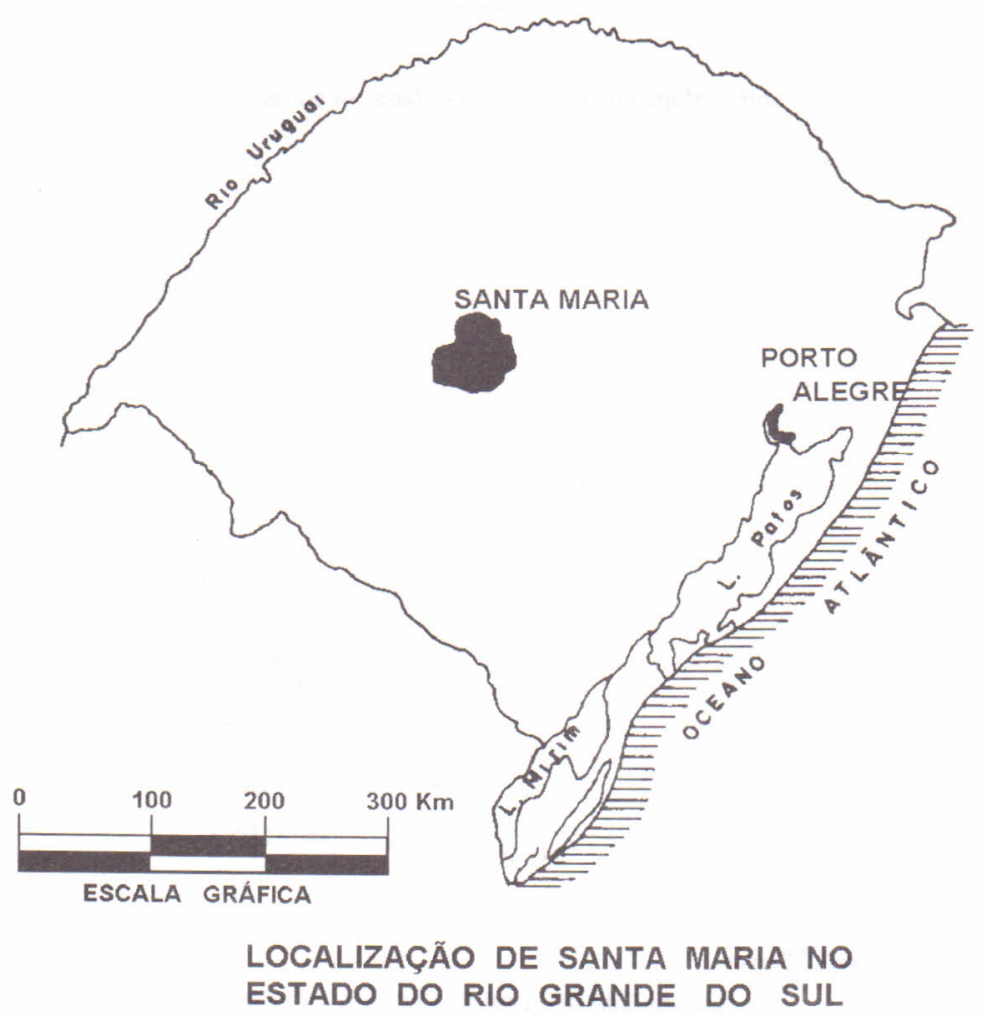

Fig. 1

Fonte: Mapa turistico de Santa Maria, 14 edição 


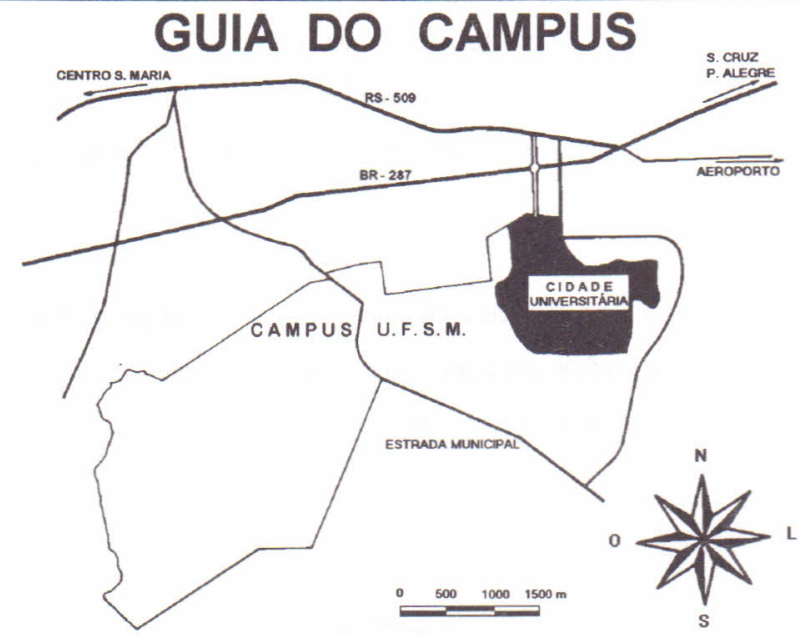

Fig. 2

Fonte: Mapa turistico de Santa Maria 14 edição

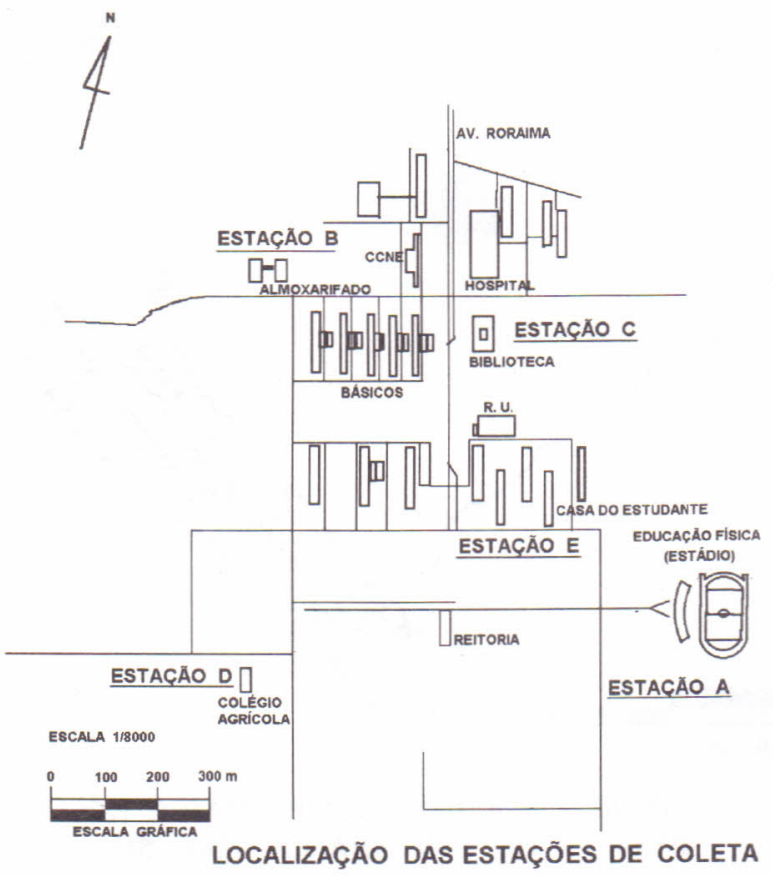

Fig. 3

Fonte: Dep. de Engenharia Rural

Setor de Fotogrametria e Fotointerpretação

Ministério da Educação

U.F.S.M. - CCR, 1991 


\section{MATERIAL E MÉTODO}

As cianoficeas estudadas foram obtidas a partir de 8 coletas, realizadas no campus da UFSM nos meses de agosto, setembro, outubro, novembro e dezembro de 1992 e janeiro, abril e junho de 1993, sem considerar a periodicidade, resultando 45 amostras.

O material coletado foi obtido do solo ou do calçamento pelo método de raspagem do substrato ou pela retirada dos fragmentos dos mesmos e, após, colocado em sacos plásticos etiquetados. Este material foi analisado ao microscópio óptico binocular Meiji no laboratório de Botânica do Departamento de Biologia do Centro de Ciências Naturais e Exatas da UFSM e preservado na forma de exsicatas.

A determinação dos gêneros foi feita com as chaves analíticas e a literatura especializada, em especial BICUDO e BICUDO (, 1970 ), COMPĖRE ( 1974 ), FRANCESCHINI ( 1983 ), ROUND ( 1983 ), TRACANNA ( 1985 ), SANT' ANNA ( 1984 ), WERNER ( 1985 ), HOFFMANN ( 1986 ) e PHUNG; COUTĖ; BOURRELLY ( 1992 ). O enquadramento taxonômico para subclasses, ordens e familias foi baseado em BOURRELLY ( 1970 ), FRANCESCHINI (1983), HOFFMANN ( 1986 ) e PHUNG; COUTĖ; BOURRELLY ( 1992 ).

\section{RESULTADOS e DISCUSSÃO}

Os 22 gêneros encontrados no material coletado podem ser determinados pela chave analitica abaixo:

\section{Ordem: CHROOCOCCALES}

1.a. Células solitárias, esféricas Synechocystis

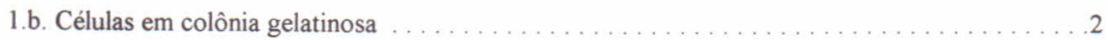

2.a. Colônia com 2 células, forma definida, bainha firme Chroococcus *

2.b. Colônia com mais de 2 células . 3 
3.a. Células ovóides ou cilindricas, envolvidas por bainha homogênea

Aphanothece

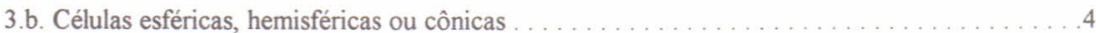

4.a. Células em grande número, distribuídas por toda a colônia e com bainha individual . . . . . . . . .

Microcystis

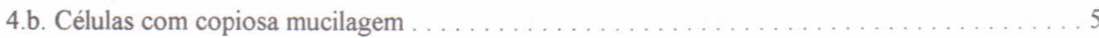

5.a. Células em número de $2-4-8$, bainha estratificada $\ldots \ldots \ldots \ldots \ldots \ldots$ Gloeocapsa

5.b. Colônia geralmente com 4 células cônicas

Myxosarcina

* No material estudado, Chroococcus sempre foi encontrado com duas células.

Ordem: NOSTOCALES (= OSCILLATORIALES)

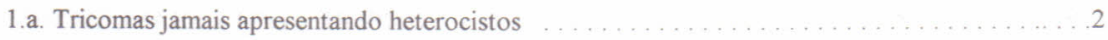

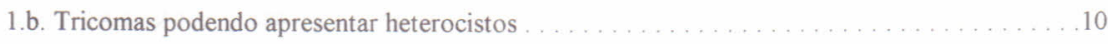

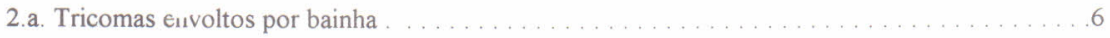

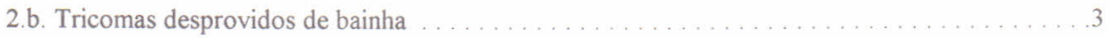

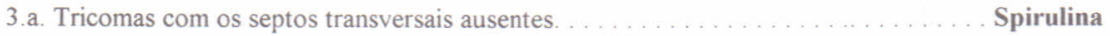

3.b. Tricomas com septos transversais visiveis $\ldots \ldots \ldots \ldots \ldots \ldots \ldots \ldots \ldots \ldots \ldots \ldots$

4.a. Tricomas com células adjacentes, não constritas $\ldots \ldots \ldots \ldots \ldots \ldots \ldots$ Oscillatoria

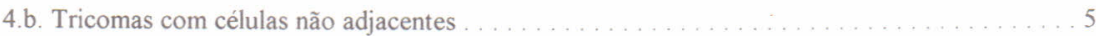

5.a. Tricomas formados por células constritas na região mediana ........... Pseudanabaena

5.b. Tricomas com células lenticulares . . . . . . . . . . . . . . Heterohormogonium

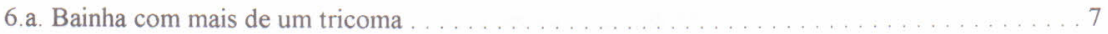

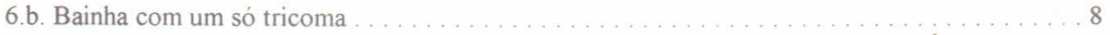

7.a. Geralmente 2 tricomas por bainha . . . . . . . . . . . . . . . . . . . . .

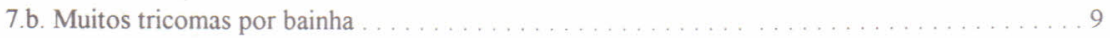

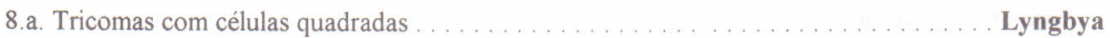

8.b. Tricomas com células mais altas que largas $\ldots \ldots \ldots \ldots \ldots \ldots$ Phormidium

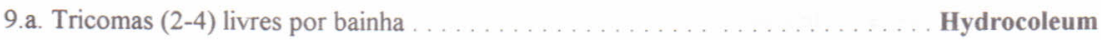

9.b. Tricomas longos na bainha . . . . . . . . . . . . . . . . . . . . . . . . . . . . . .

9.c. Pequenos tricomas na bainha $\ldots \ldots \ldots \ldots \ldots \ldots \ldots \ldots \ldots \ldots \ldots$ Lyngbyopis

10.a. Heterocistos tipicamente terminais $\ldots \ldots \ldots \ldots \ldots \ldots \ldots$ Cylindrospermum 
10.b. Tricomas com heterocistos intercalares .11

11.a. Tricomas curvos, afilados nas duas extremidades

Raphidiopsis

11.b. Tricomas nunca afilados nas extremidades. 12

12.a. Tricomas com falsas ramificações, simples ou geminadas. Scytonema

12.b. Tricomas com células em forma de barrilete

13.a. Tricomas emaranhados no interior de uma massa mucilaginosa Nostoc

13.b. Tricomas isolados. Anabaena

\section{Ordem: STIGONEMATALES}

1. Ramificações laterais irregulares, células globulares uni ou plurisseriadas

Stigonema

\section{ENQUADRAMENTO TAXONÔMICO}

Divisão: SCHIZOPHYTA ( CYANOPHYTA )

Classe: CYANOPHYCEAE

Subclasse: COCCOGONOPHYCIDEAE

Ordem: CHROOCOCCALES

Familia: CHROOCOCCACEAE

Gêneros: Chroococcus Nageli, 1849

Gloeocapsa Kutzing, 1843

Synechocystis Sauvageau, 1892

Microcystis Kutzing, 1833

Aphanothece Nageli, 1849

Myxosarcina Printz, 1921

Subclasse: HORMOGONOPHYCIDEAE

Ordem: STIGONEMATALES

Familia: STIGONEMATACEAE

Gênero: Stigonema C.A. Agardh, 1824.

Ordem: NOSTOCALES (=OSCILLATORIALES)

Familia: SCYTONEMATACEAE 
Gênero: Scytonema C.A.Agardh, 1824.

Familia: NOSTOCACEAE

Gêneros: Nostoc Vaucher, 1803.

Cylindrospermum Kutzing, 1843.

Raphydiopsis Fritsch, 1929.

Anabaena Boruu de St. Vincent, 1822.

Familia: OSCILLATORIACEAE

Gênero: Oscillatoria Vaucher, 1803.

Spirulina Turpin, 1829.

Microcoleus Desmazières, 1823.

Lyngbya C.A. Agardh, 1824.

Pseudanabaena Lauterborn, 1911.

Lyngbyopis Gardner, 1927.

Hydrocoleum Kutzing, 1843.

Schizothryx Kutzing, 1843.

Phormidium Kutzing, 1843.

Heterohormogonium Copeland, 1936.

No que se refere às famílias, o Quadro I mostra que a ordem Nostocales foi a melhor representada, registrando 3 de um total de 5 familias. As ordens Chroococcales e Stigonematales apresentaram, apenas, uma familia cada.

Analisando-se o enquadramento taxonômico e o Quadro I, verifica-se que a ordem NOSTOCALES congrega o maior número de gêneros (15), totalizando $68 \%$ dos gêneros encontrados. Esses estão distribuidos em 3 familias; a familia Oscillatoriaceae apresentou 10 gêneros do total da ordem ( Oscillatoria, Spirulina, Microcoleus, Lyngbya, Pseudanabaena, Lyngbyopis, Hydrocoleu, Schizothrix, Heterohormogonium e Phormidium ); a familia Nostocaceae apresentou 4 gêneros ( Nostoc, Cylindrospermum, Raphydiopsis e Anabaena ); a familia Scytonemataceae apresentou apenas o gênero Scytonema. A ordem CHROOCOCCALES, apresentou somente a familia Chroococcaceae com 6 gêneros somando $28 \%$ do total encontrado ( Chroococcus, Gloeocapsa, Synechocystis, Microcystis, Aphanothece e Myxosarcina ). A ordem STIGONEMATALES apresentou um só gênero (Stigonema), pertencente a família Stigonemataceae, contribuindo com $4 \%$ do total de gêneros coletados. 
QUADRO I - Número total de gêneros por familia e por ordem

\begin{tabular}{|l|c|c|}
\hline \multicolumn{1}{|c|}{ ORDENS } & NO DE FAMILIAS & No DE GÊNEROS \\
\hline CROOCOCCALES & 1 & 6 \\
\hline STIGONEMATALES & 1 & 1 \\
\hline NOSTOCALES & 3 & 15 \\
\hline TOTAL & 5 & 22 \\
\hline
\end{tabular}

O Quadro II apresenta a ocorrência dos gêneros por estação de coleta e por habitat. Verifica-se que os gêneros Chroococcus, Microcoleus, Lyngbya, Oscillatoria, Anabaena, Nostoc e Scytonema foram encontrados em todas as estações de coleta.

Os gêneros que só foram identificados em uma estação foram:

Cylindrospermum ( estação B ); Raphidiopsis e Lyngbyopsis ( estação C ); Myxosarcina e Hidrocoleum (estação E ).

A estação $C$, no mês de outubro e a estação $E$, no mês de junho, apresentaram a maior diversidade genérica. Também apresentaram uma considerável diferença em número de gêneros a favor do calçamento em relação ao solo

O gênero Cylindrospermum só foi encontrado no solo; os gêneros Myxosarcina, Hidrocoleum, Synechocystis, Aphanothece, Schizothrix, Pseudanabaena, Lingbyopsis e Raphidiopsis somente apareceram em amostras de calçamento. Os demais gêneros referidos no Quadro II ocuparam, indiferentemente, os diversos habitats.

O habitat que apresentou maior número de gêneros (20) foi o calçamento, totalizando $90 \%$ dos gêneros coletados, enquanto que no solo, foram encontrados 14 gêneros $(67 \%)$

Comparando-se as cianoficeas unicelulares com as filamentosas, verificou-se que, as unicelulares apresentaram 6 gêneros, sendo que Synechocystis é solitário e os gêneros Chroococcus, Gloeocapsa, Microcystis, Aphanothece e Myxosarcina são coloniais. As filamentosas apresentaram 16 gêneros, sendo 5 deles heterocistados ( Anabaena, Cylindrospermum, Nostoc, Scytonema e Raphidiopsis ).

Nos meses de verão, as cianoficeas não filamentosas foram pouco representadas; as filamentosas, principalmente as heterocistadas, sempre foram bem representadas.

A massa gelatinosa dos gêneros Scytonema, Microcoleus e Nostoc era bem mais copiosa nas amostras de solo do que nas amostras de calçamento. 
Quadro II: ocorrência dos gêneros por estação e por habitat

\begin{tabular}{|c|c|c|c|c|c|c|c|c|c|c|c|c|c|c|c|c|c|c|c|c|c|c|c|c|c|c|}
\hline \multirow{3}{*}{\multicolumn{2}{|c|}{$\begin{array}{l}\text { PERÍODO DE COLETA } \\
\text { ESTAÇÃO DE COLETA }\end{array}$}} & \multicolumn{11}{|c|}{1992} & \multicolumn{14}{|c|}{1993} \\
\hline & & \multirow{2}{*}{\multicolumn{2}{|c|}{$\begin{array}{l}\mathrm{AGO} \\
\mathrm{A}\end{array}$}} & \multirow{3}{*}{\begin{tabular}{|c|} 
SET \\
B \\
S \\
\end{tabular}} & \multirow{3}{*}{$\begin{array}{l}\text { OUT } \\
\text { C } \\
\text { C } \\
\end{array}$} & \multicolumn{3}{|c|}{ NOV } & \multicolumn{4}{|c|}{ DEZ } & \multicolumn{6}{|c|}{ JAN } & \multicolumn{5}{|c|}{$\mathrm{ABR}$} & \multicolumn{3}{|c|}{ JUN } \\
\hline & & & & & & \multirow{2}{*}{\begin{tabular}{|l}
$\mathrm{B}$ \\
$\mathrm{S}$ \\
\end{tabular}} & \multicolumn{2}{|c|}{ D } & \multicolumn{2}{|c|}{ A } & \multicolumn{2}{|c|}{ E } & \multicolumn{2}{|c|}{$\mathrm{A}$} & \multirow{2}{*}{\begin{tabular}{|l}
$\mathrm{B}$ \\
\end{tabular}} & \multirow{2}{*}{$\begin{array}{l}\mathrm{C} \\
\mathrm{C} \\
\end{array}$} & \multicolumn{2}{|c|}{$\mathrm{D}$} & \multicolumn{2}{|c|}{ A } & \multicolumn{2}{|c|}{\begin{tabular}{l|l}
$B$ & $C$ \\
\end{tabular}} & $\mathrm{D}$ & $\mathrm{A}$ & & $\mathrm{E}$ \\
\hline GÊNEROS & HABITAT & C & $\mathrm{S}$ & & & & $\mathrm{C}$ & $S$ & C & $S$ & $\mathrm{C}$ & $S$ & $\mathrm{C}$ & $\mathrm{S}$ & & & $\mathrm{C}$ & $\mathrm{S}$ & C & $\mathrm{S}$ & \begin{tabular}{l|l}
$S$ & \\
\end{tabular} & \begin{tabular}{l|l}
$\mathrm{C}$ & $\mathrm{C}$ \\
\end{tabular} & \begin{tabular}{l|l} 
& $S$ \\
\end{tabular} & \begin{tabular}{l|l}
$\mathrm{C}$ & $\mathrm{S}$ \\
\end{tabular} & \begin{tabular}{l|l|}
5 & $\mathrm{C}$ \\
\end{tabular} & $\mathrm{S}$ \\
\hline Chroococcus & & $\mathrm{x}$ & $x$ & $x$ & $x$ & $\mathrm{x}$ & & $\mathrm{x}$ & & $x$ & $\mathrm{x}$ & & & & & $x$ & & & $\mathrm{x}$ & $\mathrm{x}$ & \begin{tabular}{|l|} 
\\
\end{tabular} & \begin{tabular}{l|l}
$x$ & $x$ \\
\end{tabular} & \begin{tabular}{l|l}
$x$ & \\
\end{tabular} & \begin{tabular}{|l|l|}
$x$ & $x$ \\
\end{tabular} & \begin{tabular}{l|l|}
$x$ & $x$ \\
\end{tabular} & \\
\hline Synechocystis & & & & & $x$ & & & & & & & & & & & & & & & & & & & & $x$ & \\
\hline Gloeocapsa & & $\mathrm{x}$ & & & & $\mathrm{x}$ & & & & & & & & & & & & & & & & & & & $\mathrm{x}$ & \\
\hline Mixosarcina & & & & & & & & & & & & & & & & & & & & & & & & & $x$ & \\
\hline Aphanothece & & $\mathrm{x}$ & & & $\mathrm{x}$ & & & & & & & & & & & & & & & & & & & & & \\
\hline Microcoleus & & $\mathrm{x}$ & $\mathrm{x}$ & $x$ & $\mathrm{x}$ & $\mathrm{x}$ & $\mathrm{x}$ & $\mathrm{x}$ & $\mathrm{x}$ & $\mathrm{x}$ & $\mathrm{x}$ & $x$ & $x$ & $\mathrm{x}$ & $\mathrm{x}$ & $\mathrm{x}$ & $\mathrm{x}$ & $\mathrm{x}$ & $\mathrm{x}$ & $x$ & $\mathrm{x}$ & $\mathrm{x}$ & $\mathrm{x}$ & $\mathrm{x}$ & \begin{tabular}{l|l|}
$x$ & $x$ \\
\end{tabular} & $x$ \\
\hline Schizothrix & & & & & & & & & & & & & & & & & & & & & & $\mathrm{x}$ & $x$ & & \begin{tabular}{l|l}
$x$ \\
\end{tabular} & \\
\hline Hidrocoleum & & & & & & & & & & & & & & & & & & & & & & & & & $x$ & \\
\hline Lyngbya & & $x$ & $x$ & & $\mathrm{x}$ & $x$ & $x$ & $x$ & $x$ & $x$ & $x$ & $x$ & & $\mathrm{x}$ & $x$ & $\mathrm{x}$ & $\mathrm{x}$ & $\mathrm{x}$ & $\mathrm{x}$ & $\mathrm{x}$ & $\mathrm{x}$ & \begin{tabular}{l|l}
$x$ & $x$ \\
\end{tabular} & $x$ & & $x$ & $x$ \\
\hline Phormidium & & & & & & $x$ & & & & & & & & & & & & & & $\mathrm{x}$ & & & $\mathrm{x}$ & & $\mathrm{x}$ & \\
\hline Oscilatoria & & $x$ & $x$ & $x$ & $\mathrm{x}$ & $x$ & $\mathrm{x}$ & $\mathrm{x}$ & $x$ & $x$ & $\mathrm{x}$ & $x$ & $x$ & $\mathrm{x}$ & $\mathrm{x}$ & $\mathrm{x}$ & $\mathrm{x}$ & $x$ & $\mathrm{x}$ & $\mathrm{x}$ & $\mathrm{x}$ & \begin{tabular}{l|l}
$x$ & $x$ \\
\end{tabular} & $x$ & \begin{tabular}{|l|l|}
$x$ & $x$ \\
\end{tabular} & $x$ & $x$ \\
\hline Spirulina & & & & & $\mathrm{x}$ & & & $\mathrm{x}$ & & & & & & & & & & & & & & & & & & \\
\hline Cylindrospermum & & & & $x$ & & & & & & & & & & & & & & & & & & & & & & \\
\hline Anabaena & & & & & $\mathrm{x}$ & & & $x$ & & $x$ & $\mathrm{x}$ & & & & $x$ & & & & & & $x$ & $\mathrm{x}$ & $x$ & & $x$ & \\
\hline Nostoc & & $x$ & $x$ & $x$ & $\mathrm{x}$ & $x$ & $\mathrm{x}$ & $\mathrm{x}$ & $x$ & $x$ & $\mathrm{x}$ & & & $\mathrm{x}$ & $x$ & $\mathrm{x}$ & $x$ & & $\mathrm{x}$ & $\mathrm{x}$ & $\mathrm{x}$ & \begin{tabular}{l|l}
$x$ & $x$ \\
\end{tabular} & $x$ & $x$ & $x$ & \\
\hline Raphidiopsis & & & & & $\mathrm{x}$ & & & & & & & & & & & & & & & & & & & & & \\
\hline Scytonema & & $\mathrm{x}$ & $x$ & $x$ & $x$ & $x$ & $\mathrm{x}$ & $x$ & $\mathrm{x}$ & $\mathrm{x}$ & $x$ & $x$ & $\mathrm{x}$ & $\mathrm{x}$ & $x$ & $\mathrm{x}$ & $\mathrm{x}$ & $x$ & $\mathrm{x}$ & $\mathrm{x}$ & $x$ & \begin{tabular}{l|l}
$\mathrm{x}$ & $x$ \\
\end{tabular} & $x$ & $x$ & \begin{tabular}{|l|l|}
$x$ & $x$ \\
\end{tabular} & $x$ \\
\hline Stigonema & & & $\mathrm{x}$ & & & & & & & $\mathrm{x}$ & & & & $\mathrm{x}$ & $\mathrm{x}$ & & & & & $\mathrm{x}$ & $\mathrm{x}$ & $\mathrm{x}$ & & & \begin{tabular}{l|l|}
$x$ & $x$ \\
\end{tabular} & \\
\hline Pseudoanabaena & & & & & $x$ & & & & & & $\mathrm{x}$ & & & & & $\mathrm{x}$ & & & & & & & & & & \\
\hline Microsystis & & $\mathrm{x}$ & & $x$ & & & & & & & & & & & & & & & & & & & & & & \\
\hline 73 & & & & $\mathrm{x}$ & & & $\mathrm{x}$ & & & & & & & & & & & & & & & $\mathrm{x}$ & $x$ & & & \\
\hline Lyngbyopis & & & & & $x$ & & & & & & & & & & & & & & & & & & & & & \\
\hline $\mathrm{N}^{\circ}$ gêneros/habitat & & 9 & 7 & 8 & 13 & 9 & 5 & 8 & 5 & 8 & 8 & 4 & 3 & 6 & 7 & 8 & 5 & 4 & 6 & 8 & 8 & \begin{tabular}{l|l}
8 & 8 \\
\end{tabular} & \begin{tabular}{|l|l|}
8 & 5 \\
\end{tabular} & & & 4 \\
\hline $\mathrm{N}^{\circ}$ gêneros/estação & & & 10 & 8 & 13 & 9 & 8 & & 8 & & 8 & & 6 & & 7 & 8 & 5 & & 8 & & 8 & \begin{tabular}{c|c}
8 \\
\end{tabular} & 9 & & \begin{tabular}{l|l}
7 & 14 \\
\end{tabular} & \\
\hline $\mathrm{N}^{\circ}$ gêneros/coleta & & & 10 & 8 & 13 & & 11 & & & 9 & & & & & 9 & & & & & 11 & 1 & & & & 14 & \\
\hline
\end{tabular}

Legenda: C - calçamento S - solo 


\section{CONCLUSÕES}

No levantamento de gêneros de cianoficeas de solo e calçamento do Campus da Universidade Federal de Santa Maria foram identificados 22 gêneros distribuídos em 5 familias e 3 ordens, o que, apesar do pouco número de coletas, evidencia uma flora rica em cianoficeas.

A abundância de representantes dos gêneros Microcoleus, Oscillatoria e Scytonema, que foram encontrados em todas as estações de coleta, poderia indicar sua capacidade de adaptação, tanto em solo quanto em calçamento.

Pôde-se observar que os gêneros Oscillatoria, Lyngbya, Phormidium, Microcoleus, Scytonema e Nostoc apresentavam maior diversidade de espécies em amostras de solo do que em calçamento, o que sugere que, a umidade é um fator importante para o ciclo biológico dessas algas.

Observações visuais confirmaram SANT'ANNA ( 1984 ) que já havia observado que os gêneros heterocistados Nostoc, Scytonema e Cylindrospermum formam uma massa macroscópica que se conserva por mais tempo devido a presença de células de resistência ( heterocistos ).

Dos 22 gêneros encontrados Anabaena e Nostoc já foram citados em solo brasileiro nos trabalhos de LYRA ( 1972 ), OLIVEIRA ( 1980 ) e AZEVEDO ( 1991 ). Os demais gêneros foram citados ou por um ou por no máximo dois desses autores.

\section{BIBLIOGRAFIA}

AZEVEDO, M.T.P. Edaphic blue-green algas fron the São Paulo Botanical Garden, Brazil. Stuttgart: Algological Studies 64, 503-526. 1991.

BERTAGNOLLI, M. P. Levantamento de Gêneros de cianofíceas de solo e calçamento no Campus da Universidade Federal de Santa Maria, Rio Grande do Sul, Brasil. Santa Maria Monografia de Epecialização, 1994. 58 p.

BICUDO, C.E.M., BICUDO, R.M.T. Algas de águas continentais brasileiras; chave ilustrada para identificação de gêneros. São Paulo: EDUSP, 1970. 228 p.

BOURRELLY, P. Les algues d' eau douce; Initiation à la Sistematique. Les algues bleues et rouges. Paris, N. Boubee, v.3, 1970. $512 \mathrm{p}$.

COMPÈRE, P. Algues De La Région Du Lac Tchad. II Cyanophycées, Cah. O.R. S T. O. M. Sér Hydrobiol, Vol VIII n. 314, 165-199, 1974.

DANIN, A., BAR - OR, Y., DOR I. e YBRAELI, T. The role of Cyanobacteria in stabilization of sand dunes in souther Israel. Ecolmediterr 15 (1/2): 15 - 64, 1989.

DAVEY, M., DAVISON H.P.B., RICHARD K. J. e WYNN - WILLIAMS D. D. Attachment 
and growth of ntartic soil cyanobacteria and algae on natural and artificial substrato. Inglaterra: Soil Biol Biochem 23 (2): 185 - 192, 1991.

FRANCESCHINI, I. M. Levantamento das Nostocophyceae do Rio Seco, Torres, Rio Grande Sul, Brasil. Tese (Mestrado em Ciências Biológicas) Instituto de Biociências UFRGS Porto Alegre: 1983. 157 p.

HOFFMANN, L. Cyanophycées aériennes et subaériennes du Grand-Duché de Luxembourg. Bull. Jard. Bot. Nat. Belg: Bull Natt, Plantentuin Belg. 56 (1/2) 77-127, 30-6, 1986.

KOSTYKOV, I. Algae of rarines and their autierosion role. Ukr. Bot. Zh 47 (1): 43 - 47, 1990.

LYRA, E. Algas no solo do agreste de Pernambuco. U. F. de Pernambuco: Publicação n. 693: $1-8,1972$.

OLIVEIRA, M. S. B., OLIVEIRA, N. A., DAINESI, M. B., CARDOSO, E. J. B. N. Ocorrência de Algas Cianoficeas em dois solos do Municipio de Piracicaba. São Paulo: O solo, 72, 62-64, 1980 .

ORTEGA - CALLO J. J., HERNANDEZ - MARINE M., SAIZ - JIMENEZ C. Biodeterioration of building materials by Cyanobacteria and algae. Espanha: Int Biodeterior $28(1-4): 165$ $-186,1991$

PEREIRA, R .B., GARCIA NETO, L. R., BORIN, C. J. A., BARROS SARTORI, M. G. Contribuição à Geografia Física do Município de Santa Maria: Unidades de Paisagem. Santa Maria: Geografia - Ensino \& Pesquisa, 3: 37 - 68, Dez 1989. 175 p

PHUNG, T. N. H., COUTÉ, A., BOURRELly, P. Les Cyanophycées du delta du Mékong (Viet -Nan). Stuttgart: Nova Hedwigia 54, 3- 4, 403-446. 1992.

ROUND, F. E. Biologia das Algas. 2 ed. Rio de janeiro: Guanabara Dois, 1983. 263 p

SANT 'ANNA, C. L. Flora de Cyanophyceae associada a Briófita, Município de Campina Verde, M. G. Rickia 11: 129-142, 1984.

SENNA, P. A. C. Nostocoficeas do Município de São Paulo, Estado de São Paulo, Brasil. São Paulo: 1982.249 p. Dissertação de mestrado.

SHI - MEI, L., SHANG - HOO, L. Interaction of two populations of the blue-green algae in coculture. China: Acta Bot Sin 33 (2): 110-117, 1991.

TRACANNA, B. C. Algas del Noroeste Argentino (Excluyendo las Diatomophyceae). San Miguel De Tucumán: Opera Lilloano 35, 1985. $136 \mathrm{p}$

WERNER, V. R. Cianoficeas planctônicas da Lagoa de Tramandai e da Lagoa do Armazém, Rio Grande do Sul, Brasil. Iheringia, Sér. Bot., Porto Alegre (37): 33-70, 1988. 